\title{
A Simple yet Effective Joint Training Method for Cross-Lingual Universal Dependency Parsing
}

\author{
Danlu Chen*1, Mengxiao Lin*12, Zhifeng Hu*1, Xipeng Qiu ${ }^{1}$ \\ ${ }^{1}$ Fudan University \\ ${ }^{2}$ Megvii Inc \\ danluchen@fb.com, linmengxiao@megvii.com, \{zfhu16,xipeng\}@fudan.edu.cn
}

\begin{abstract}
This paper describes Fudan's submission to CoNLL 2018's shared task Universal Dependency Parsing. We jointly train models when two languages are similar according to linguistic typology and then do an ensemble of the models using a simple re-parse algorithm. Our system outperforms the baseline method by $4.4 \%$ and $2.1 \%$ on the development and test set of CoNLL 2018 UD Shared Task, separately. ${ }^{1}$. Our code is available on https://github.com/ taineleau/FudanParser.
\end{abstract}

\section{Introduction}

Dependency Parsing has been a fundamental task in Natural Language Processing (NLP). Recently, universal dependency parsing (Zeman et al., 2018a,b; Nivre et al., 2018) has unified the annotations of different languages and thus made transfer learning among languages possible. Several works using cross-lingual embedding (Duong et al., 2015; Guo et al., 2015) have successfully increased the accuracy of cross-lingual parsing. Beyond embedding-based methods, a natural question is whether we can use a simple way to utilize the universal information. Some previous research either regarded the universal information as extra training signals (e.g., delexicalized embedding (Dehouck and Denis, 2017)), or implicitly trained a network with all features (e.g., adversarial training for parsing in Sato et al. (2017)). In our system, we manually and explicitly share the universal annotations via a shared LSTM component.

\footnotetext{
* Authors contributed equally.

${ }^{1}$ Unfortunately, we did not finish the run before the deadline. As a result, the official accuracy gain for test set is only $0.54 \%$ and we ranks 17 th out of 27 teams.
}

Similar to Vania et al. (2017), different languages are first grouped based on typology, as shown in table 1 . Then, we train a shared model for each pair of languages within the same group, and apply a simple ensemble method over all trained models. Note that our method is orthogonal to other cross-lingual approaches for universal parsing such as cross-lingual embedding.

In the following parts, we first describe the baseline method (Section 2) and our system (Section 3). We show the result on both development set and test set in Section 4 and provide some analysis of the model in Section 5.

\section{Baseline}

In this section, we briefly introduce the baseline system, UDPipe 1.2 (Straka and Straková, 2017), which is an improved version of original UDPipe (Straka et al., 2016). The tokenizing, POS tagging and lemma outputs of UDPipe are utilized by $\mathrm{Fu}$ danParser.

UDPipe employs a GRU network during the inference of segmentation and tokenization. The tagger uses characters features to predict the POS and lemma tags. Finally, a transition-based neural dependency parser with one hidden layer predicts the transition actions. The parser also makes use of the information from lemmas, POS taggings and dependency relationships through a group of embeddings precomputed by word2vec.

In the later discussion, we take the baseline performance result from the web page of the shared task $^{2}$ for comparison.

\section{System Description}

In this submission, we only consider parsing in an end-to-end manner and handle each treebank sep-

\footnotetext{
${ }^{2}$ http: / / universaldependencies.org/ conll18/baseline.html
} 


\begin{tabular}{|c|c|}
\hline Group & Datasets \\
\hline \multirow[t]{8}{*}{ germanic } & Afrikaans-AfriBooms Danish- \\
\hline & DDT Dutch-Alpino Dutch- \\
\hline & LassySmall English-EWT \\
\hline & English-GUM \\
\hline & LinES German-GSD Gothic- \\
\hline & PROIEL Norwegian-Bokmaal \\
\hline & Norwegian-Nynorsk Swedish- \\
\hline & LinES Swedish-Talbanken \\
\hline \multirow[t]{2}{*}{ indo-iranian } & 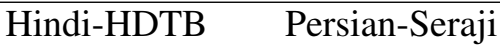 \\
\hline & Urdu-UDTB \\
\hline \multirow[t]{2}{*}{ latin } & Latin-PROIEL \\
\hline & Latvian-LVTB \\
\hline \multirow[t]{7}{*}{ romance } & Catalan-AnCora $\quad$ French-GSD \\
\hline & French-Sequoia French-Spoken \\
\hline & Galician-CTG Italian-ISDT \\
\hline & Italian-PoSTWITA Old_French- \\
\hline & SRCMF Portuguese-Bosque \\
\hline & Romanian-RRT Spanish- \\
\hline & AnCora \\
\hline semitic & Arabic-PADT Hebrew-HTB \\
\hline \multirow[t]{7}{*}{ slavic } & $\begin{array}{ll}\text { Bulgarian-BTB } & \text { Croatian- }\end{array}$ \\
\hline & SET Czech-CAC Czech- \\
\hline & FicTree $\quad$ Czech-PDT \\
\hline & Old_Church_Slavonic-PROIEL \\
\hline & Polish-LFG Polish-SZ Russian- \\
\hline & SynTagRus $\quad$ Serbian-SET \\
\hline & Slovak-SNK Slovenian-SSJ \\
\hline \multirow[t]{2}{*}{ turkish } & Turkish-IMST Ukrainian-IU \\
\hline & Uyghur-UDT \\
\hline \multirow[t]{2}{*}{ uralic } & Estonian-EDT \\
\hline & Finnish-TDT \\
\hline
\end{tabular}

Table 1: Grouping languages according to typology. arately. We first train a monotonic model for all "big" treebanks. Besides, for each language, there are $N-1$ models fine-tuned from joint-trained (see Figure 2), where $N$ is the number of languages in the same language group.

For small treebanks where training set is less than 50 sentences, we use the delexicalized method the same as Shi et al. (2017)'s approach for the surprise languages. Shi et al. (2017) took delexicalized features (morphology and POS tag) as input and apply 50\% dropout rate to the input. In practice, we found that the baseline method performs much better than ours on "fi_pud", "br_keb" "ja_modern" and "th_pud", so we use the baseline method instead for these languages.

Our whole system needs about 90 hours to do the inference of all models on TIRA and requires no more than $560 \mathrm{M}$ main memory.

\subsection{Architecture}

Features We use words, characters as the lexical information, and use morphological features ${ }^{3}$ and POS tags as the delexicalized information. We also tried subword embeddings, but it mostly did not help. More precisely, the character-level features are treated as bag-of-characters. Similarly, we use bag-of-morphology for morphological features (one can see number=single as a character). We first assign the embedding vectors for characters and morphological features, and then for each word, we apply a Convolutional Network (CNN) to encode variable length embeddings into one fixed length feature.

Biaffine BiLSTM. Similar to Shi et al. (2017); Sato et al. (2017); Vania et al. (2017), we use last year's first-place model (Dozat et al., 2017), the graph-based biaffine bizLSTM model as our backbone. Given a sentence of $N$ words, the input is first fed to a bi-directional LSTM and obtain the feature of each word $w_{i}$. A head MLP and a dependent MLP are used to translate the features, which is then fed into a hidden layer to calculate the biaffine attention. Finally, we are able to compute the score of arcs and labels in following way:

\footnotetext{
${ }^{3}$ we take the features column of the UD data as the morphological features, which includes case, number, tense, mood and so on. See http: //universaldependencies.org/u/feat/ index. html for detailed information.
} 


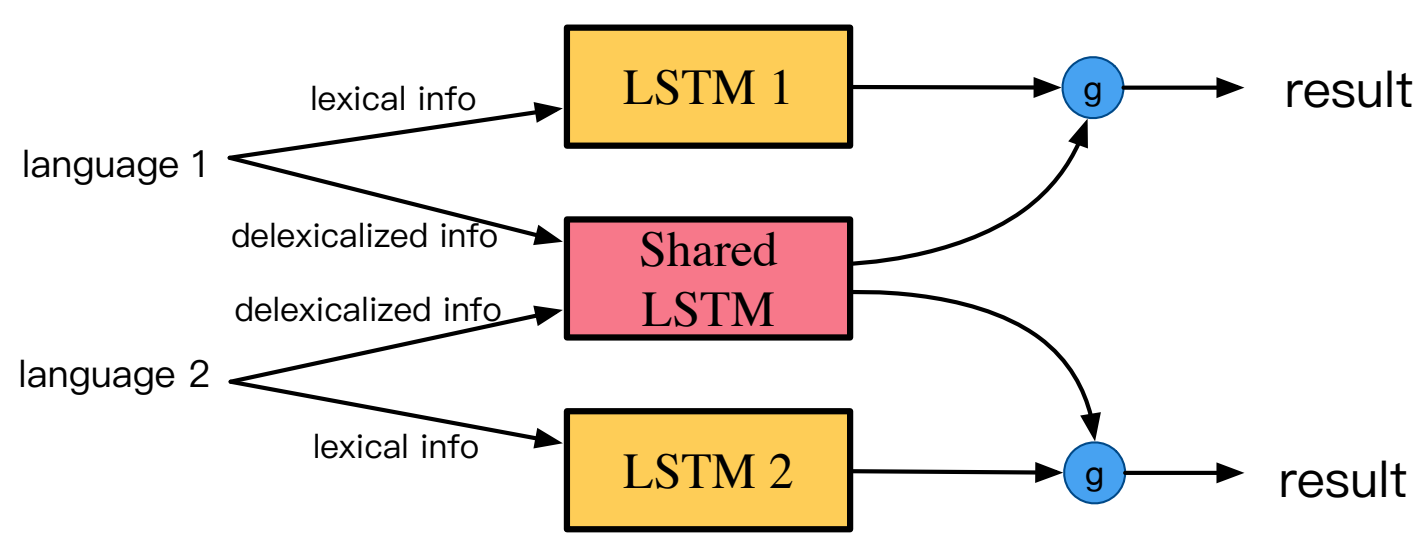

Figure 1: An illustration of the joint training framework for two languages.

$$
\begin{aligned}
h_{i}^{h} & =\operatorname{MLP}_{\text {head }}\left(w_{i}\right) \\
h_{i}^{d} & =\operatorname{MLP}_{d e p}\left(w_{i}\right) \\
s_{i} & =H^{h} U_{1} h_{i}^{d}+H^{h} u_{2}
\end{aligned}
$$

where $U_{1} \in \mathbb{R}^{d \times d}$ and $u_{2} \in \mathbb{R}^{d}$ are trainable parameters.

\subsection{Joint Training}

For a joint training model of $N$ languages, we have $N+1$ Biaffne Bi-LSTMs (called LSTMs), see Figure 1. For each language, we have a languagespecific LSTM to process the lexical information such as word- or character- level embedding, and the output is $w_{i, j}^{l}$. For all languages we have a shared LSTM which takes delexicalized information such as morphology and POS tags as input and the output is $w_{i, j}^{d}$. Inspired by Sato et al. (2017), we use a gating mechanism to combine these two set of features. Formally,

$$
\begin{gathered}
x=\left[w_{i, j}^{l} ; w_{i, j}^{d}\right], \\
g=G(x), y=x \odot g,
\end{gathered}
$$

where $w^{l}$ indicates lexical feature, $w^{d}$ indicates delexicalized feature, and $\odot$ is element-wise multiplication.

The difference between Sato et al. (2017) and ours is that we remove the adversarial training loss, which is because we have already use the universal information in the shared network.

\subsection{Fine-tuning}

We fine-tunning each joint-training model for 100 steps (see Figure 2).

\subsection{Tree Ensemble}

We follow the re-parsing method proposed in Sagae and Lavie (2006) to perform model ensemble. Suppose $k$ parsing trees have been obtained, denoted by $T_{1}, T_{2}, \ldots T_{k}$, a new graph is constructed by setting the score of each edge to

$$
S[u \rightarrow v]=\sum_{i=1}^{k}[u \rightarrow v] \in T_{k}
$$

This graph is feed to a MST algorithm to get the ensemble parsing tree $T_{e}$. Then the relation label of edge $[u \rightarrow v]$ in $T_{e}$ is voted by all inputs $T_{i}$ that contains edge $[u \rightarrow v]$.

\subsection{Hyper-parameters}

We followed the hyper-parameter settings in (Dozat et al., 2017). We train 30,000 steps for each model and then fine-tune (onot necessary) for 100 steps for the given language. For all the input features, the dimension is 100. For LSTM, we use hidden size equals to 400 and the number of layers is 3. $0.33 \%$ dropout rate is applied to the input and LSTM hidden layer. We use Bayesian dropout (Gal and Ghahramani, 2016) in the LSTM layers. We also use word dropout (dropping the whole word with a probability) in the input layer.

\section{Results}

The results of the test and development set are shown in Table 5 and Table 6, respectively. The first three columns are the baseline results and the second three columns are the results of our submission. Also, we list the performance improvement of Fudan Parser compared to the baseline system in the last three columns. 

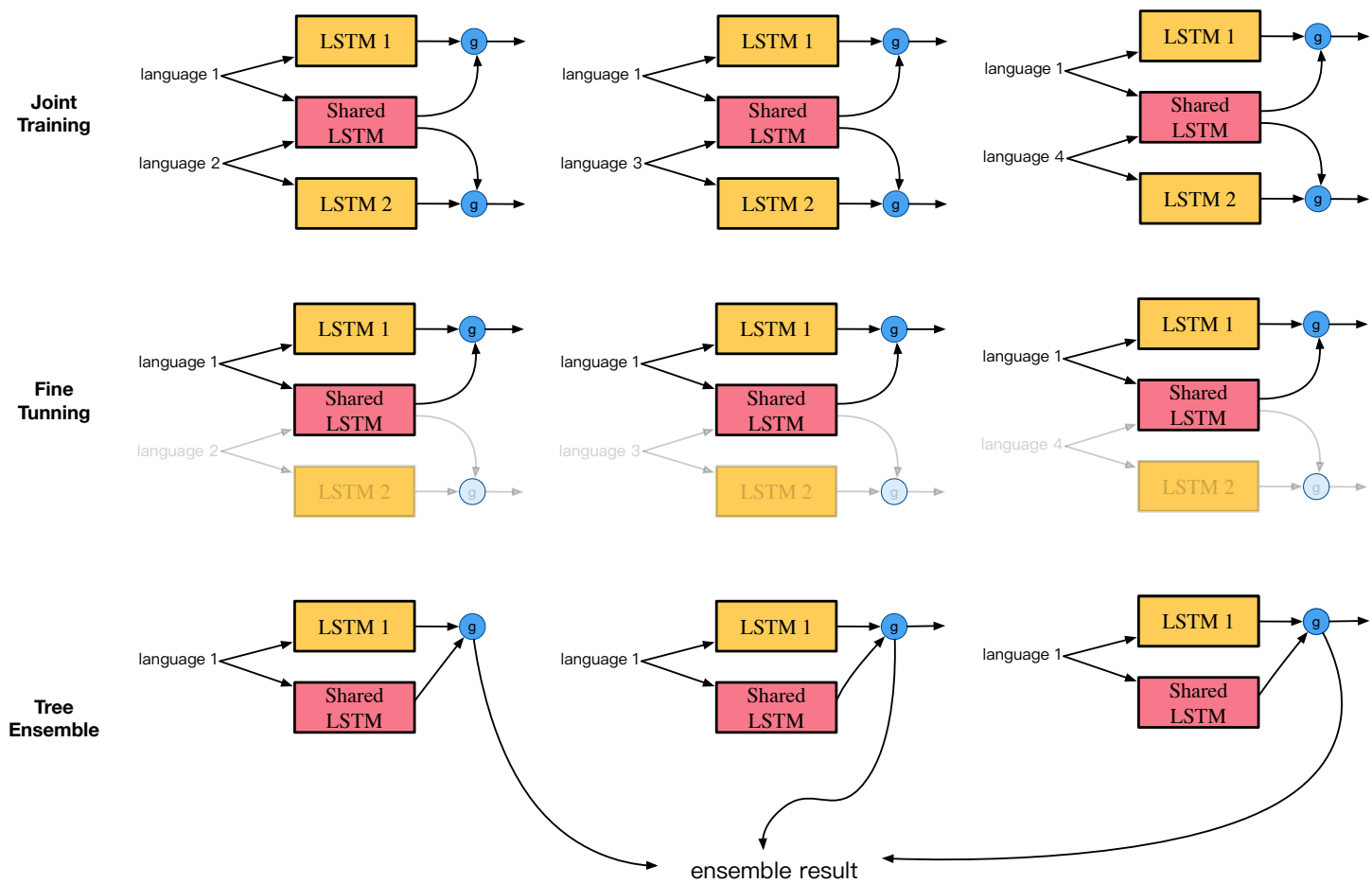

Figure 2: Take four languages as an example. We aim at testing sentence in language 1. We first jointly train languages 1 and other three languages in three separate network. And then we only keep LSTM 1 and the shared LSTM part to fine tune the models for language 1. Finally we re-parse it as an ensemble to obtain the final parsing tree for a given sentence in language 1 .

As shown in both Table 6 and 6, we find that our system achieves higher improvements on the datasets with large size of training data. It is reasonable since our model contains enormous parameters, which is easy to get overfitting if the training set is too small. More analysis are included in Section 5.

\section{Analysis}

\subsection{Language similarity}

The accuracy of the joint training model actually reveals the syntactic similarity between two languages. The accuracy of three language groups, Slavic (Table 2), Romance (Table 3) and Germanic (Table 4). A number in row $i$, column $j$ means the accuracy of language $i$ testing on the model jointly training on language $i$ and language $j$. The bold font indicates it is the best model for language $i$. We can see that for every language, jointly trained models consistently beat single models (the number on the diagonal) which shows the efficacy of the proposed approach.

\subsection{Morphology}

Morphology is extremely helpful when predicting the dependency between words, especially for those morphology rich languages. However, the UD Parsing task is not done in an end-to-end fashion (i.e. the input morphological features are not the ground-true labels) and thus the morphology information is noisy. The performance is hurt greatly because of the noisy predicted morphology features. A significant accuracy gain should be obtained if a better morphology prediction model is used.

\section{Conclusion}

Our system provided a simple yet effective method -sharing the universal features to the same part of neural network- to boost the accuracy of syntactic parsing. We also demonstrated that morphological feature plays an important role in syntactic parsing, which is a promising direction to work on.

In the future, we can investigate a better way to do the ensemble or apply a multi-model compression method (e.g. knowledge distillation) to reduce the computational cost. Also, we can explore 
Table 2: Slavic languages joint training result.

\begin{tabular}{r|lllllll|l} 
Acc. $(\%)$ & bg & hr & cs & pl & ru & sk & sl & max improvement \\
\hline bg & 92.6 & 92.5 & $\mathbf{9 2 . 8}$ & 92.7 & 92.7 & 92.3 & 92.4 & 0.2 \\
hr & 85.7 & 86 & $\mathbf{8 6 . 1}$ & 85.2 & 85.5 & 85.8 & 85.5 & 0.1 \\
cs & 91.2 & 91.2 & 91.2 & 91.1 & $\mathbf{9 1 . 3}$ & 91.3 & 91.2 & 0.1 \\
pl & $\mathbf{9 0 . 4}$ & 89.8 & 90.2 & 90.1 & 90.2 & 90.4 & 90.8 & 0.3 \\
ru & 84.4 & 84.7 & $\mathbf{8 5 . 2}$ & 84.4 & 83.8 & 84.1 & 84.6 & 1.4 \\
sk & 86.4 & 86.2 & $\mathbf{8 7 . 8}$ & 85.9 & 86.4 & 86.7 & 86.1 & 1.1 \\
sl & 91.4 & $\mathbf{9 1 . 8}$ & 91.7 & 91.4 & 91.4 & 91 & 91.2 & 0.6 \\
Avg. & & & & & & & & 0.54 \\
\hline \# samples & 8908 & 7690 & 68496 & 6101 & 3851 & 8484 & 6479 &
\end{tabular}

Table 3: Romance languages joint training result.

\begin{tabular}{l|lllllll|l} 
Acc. (\%) & ca & fr & gl & it & pt & ro & es & max improvement \\
\hline ca & 92.6 & 92.4 & 92.6 & $\mathbf{9 2 . 7}$ & 92.6 & 92.4 & 92.5 & 0.1 \\
fr & 93.1 & 92.9 & 93 & $\mathbf{9 3 . 4}$ & 93.2 & 93.1 & 93.2 & 0.5 \\
gl & $\mathbf{8 6 . 9}$ & 86.3 & 86.1 & 86.7 & 86.4 & 86.3 & 86.4 & 0.8 \\
it & 93 & 92.6 & 92.7 & 92.3 & 93 & 92.8 & $\mathbf{9 3 . 1}$ & 0.8 \\
pt & $\mathbf{9 3}$ & 92.8 & 92.7 & 92.8 & 92.6 & 92.9 & 92.8 & 0.4 \\
ro & 88.8 & 88.9 & 88.9 & $\mathbf{8 9}$ & 88.7 & 88.4 & 88.7 & 0.6 \\
es & 90.9 & 90.8 & 90.5 & $\mathbf{9 1 . 1}$ & 91 & 90.6 & 90.7 & 0.4 \\
Avg. & & & & & & & & 0.56 \\
\hline \# samples & 13124 & 14554 & 2277 & 12839 & 8332 & 8044 & 14188 &
\end{tabular}

Table 4: Germanic languages joint training result.

\begin{tabular}{l|lllll|l} 
Acc. $(\%)$ & da & nl & en & de & sv & max improvement \\
\hline da & 85 & 85.1 & 85 & 85.2 & $\mathbf{8 5 . 6}$ & 0.6 \\
nl & 89 & 88.8 & 88.7 & $\mathbf{8 9 . 3}$ & 88.6 & 0.5 \\
en & $\mathbf{8 9 . 4}$ & 89.1 & 88.9 & 88.9 & 89.1 & 0.5 \\
de & 88.5 & $\mathbf{8 8 . 9}$ & 88.7 & 88.6 & 88.5 & 0.3 \\
sv & 85.7 & 85 & $\mathbf{8 6 . 5}$ & 85.9 & 85 & 1.5 \\
Avg. & & & & & & 0.68 \\
\hline \# samples & 4384 & 12331 & 12544 & 14119 & 4303 &
\end{tabular}

a more sophisticated model (e.g., Neural Architecture Search (Zoph and Le, 2016)) for joint training on multiple languages. 


\begin{tabular}{|c|c|c|c|c|c|c|c|c|c|}
\hline & \multicolumn{3}{|c|}{ Baseline } & \multicolumn{3}{|c|}{ Fudan } & & mprovem & \\
\hline Language code & LAS & MLAS & BLEX & LAS & MLAS & BLEX & LAS & MLAS & BLEX \\
\hline af_afribooms & $77.88 \%$ & $64.48 \%$ & $66.60 \%$ & $80.02 \%$ & $67.34 \%$ & $66.04 \%$ & $2.14 \%$ & $2.86 \%$ & $-0.56 \%$ \\
\hline grc_perseus & $57.75 \%$ & $31.05 \%$ & $38.74 \%$ & $63.31 \%$ & $34.58 \%$ & $38.22 \%$ & $5.56 \%$ & $3.53 \%$ & $-0.52 \%$ \\
\hline grc_proiel & $67.57 \%$ & $49.51 \%$ & $55.85 \%$ & $69.54 \%$ & $51.35 \%$ & $53.03 \%$ & $1.97 \%$ & $1.84 \%$ & $-2.82 \%$ \\
\hline ar_padt & $66.41 \%$ & $55.01 \%$ & $57.60 \%$ & $67.33 \%$ & $55.88 \%$ & $58.63 \%$ & $0.92 \%$ & $0.87 \%$ & $1.03 \%$ \\
\hline hy_armtdp & $21.79 \%$ & $5.00 \%$ & $11.94 \%$ & $26.24 \%$ & $10.00 \%$ & $13.85 \%$ & $4.45 \%$ & $5.00 \%$ & $1.91 \%$ \\
\hline eu_bdt & $70.13 \%$ & $57.65 \%$ & $63.50 \%$ & $72.74 \%$ & $58.98 \%$ & $57.41 \%$ & $2.61 \%$ & $1.33 \%$ & $-6.09 \%$ \\
\hline br_keb & $10.25 \%$ & $0.00 \%$ & $0.00 \%$ & $10.25 \%$ & $0.00 \%$ & $0.00 \%$ & $0.00 \%$ & $0.00 \%$ & $0.00 \%$ \\
\hline bg_btb & $84.91 \%$ & $75.30 \%$ & $73.78 \%$ & $86.47 \%$ & $77.04 \%$ & $77.70 \%$ & $1.56 \%$ & $1.74 \%$ & $3.92 \%$ \\
\hline bxr_bdt & $12.61 \%$ & $0.00 \%$ & $5.00 \%$ & $12.61 \%$ & $0.00 \%$ & $5.00 \%$ & $0.00 \%$ & $0.00 \%$ & $0.00 \%$ \\
\hline ca_ancora & $85.61 \%$ & $76.74 \%$ & $77.27 \%$ & $88.37 \%$ & $80.17 \%$ & $66.01 \%$ & $2.76 \%$ & $3.43 \%$ & $-11.26 \%$ \\
\hline hr_set & $78.61 \%$ & $58.72 \%$ & $70.26 \%$ & $81.01 \%$ & $60.92 \%$ & $66.61 \%$ & $2.40 \%$ & $2.20 \%$ & $-3.65 \%$ \\
\hline cs_cac & $83.72 \%$ & $70.89 \%$ & $77.65 \%$ & $86.28 \%$ & $73.90 \%$ & $79.54 \%$ & $2.56 \%$ & $3.01 \%$ & $1.89 \%$ \\
\hline cs_fictree & $82.49 \%$ & $69.26 \%$ & $74.96 \%$ & $85.22 \%$ & $72.01 \%$ & $77.18 \%$ & $2.73 \%$ & $2.75 \%$ & $2.22 \%$ \\
\hline cs_pdt & $83.94 \%$ & $74.32 \%$ & $79.39 \%$ & $85.35 \%$ & $75.89 \%$ & $74.68 \%$ & $1.41 \%$ & $1.57 \%$ & $-4.71 \%$ \\
\hline cs_pud & $80.08 \%$ & $66.53 \%$ & $73.79 \%$ & $81.05 \%$ & $67.97 \%$ & $68.99 \%$ & $0.97 \%$ & $1.44 \%$ & $-4.80 \%$ \\
\hline da_ddt & $75.43 \%$ & $65.41 \%$ & $66.04 \%$ & $78.38 \%$ & $68.20 \%$ & $62.51 \%$ & $2.95 \%$ & $2.79 \%$ & $-3.53 \%$ \\
\hline nl_alpino & $77.60 \%$ & $61.55 \%$ & $64.76 \%$ & $79.02 \%$ & $63.89 \%$ & $60.53 \%$ & $1.42 \%$ & $2.34 \%$ & $-4.23 \%$ \\
\hline nl_lassysmall & $74.56 \%$ & $61.85 \%$ & $63.14 \%$ & $77.41 \%$ & $64.64 \%$ & $49.81 \%$ & $2.85 \%$ & $2.79 \%$ & $-13.33 \%$ \\
\hline en_ewt & $77.56 \%$ & $68.70 \%$ & $71.02 \%$ & $78.44 \%$ & $68.99 \%$ & $62.92 \%$ & $0.88 \%$ & $0.29 \%$ & $-8.10 \%$ \\
\hline en_gum & $74.20 \%$ & $62.66 \%$ & $62.14 \%$ & $75.29 \%$ & $63.36 \%$ & $57.45 \%$ & $1.09 \%$ & $0.70 \%$ & $-4.69 \%$ \\
\hline en_lines & $73.10 \%$ & $64.03 \%$ & $65.42 \%$ & $74.83 \%$ & $65.78 \%$ & $62.80 \%$ & $1.73 \%$ & $1.75 \%$ & $-2.62 \%$ \\
\hline en_pud & $79.56 \%$ & $67.59 \%$ & $71.14 \%$ & $78.80 \%$ & $67.20 \%$ & $64.26 \%$ & $-0.76 \%$ & $-0.39 \%$ & $-6.88 \%$ \\
\hline et_edt & $75.02 \%$ & $67.12 \%$ & $63.85 \%$ & $77.80 \%$ & $69.82 \%$ & $61.53 \%$ & $2.78 \%$ & $2.70 \%$ & $-2.32 \%$ \\
\hline fo_oft & $25.19 \%$ & $0.00 \%$ & $5.00 \%$ & $26.95 \%$ & $0.00 \%$ & $5.00 \%$ & $1.76 \%$ & $0.00 \%$ & $0.00 \%$ \\
\hline fi_ftb & $75.64 \%$ & $65.22 \%$ & $61.76 \%$ & $78.27 \%$ & $68.03 \%$ & $66.99 \%$ & $2.63 \%$ & $2.81 \%$ & $5.23 \%$ \\
\hline fi_pud & $80.15 \%$ & $73.16 \%$ & $65.46 \%$ & $80.15 \%$ & $73.16 \%$ & $65.46 \%$ & $0.00 \%$ & $0.00 \%$ & $0.00 \%$ \\
\hline fi_tdt & $76.45 \%$ & $68.58 \%$ & $62.19 \%$ & $79.18 \%$ & $70.74 \%$ & $59.79 \%$ & $2.73 \%$ & $2.16 \%$ & $-2.40 \%$ \\
\hline fr_gsd & $81.05 \%$ & $72.16 \%$ & $74.22 \%$ & $83.19 \%$ & $74.01 \%$ & $68.58 \%$ & $2.14 \%$ & $1.85 \%$ & $-5.64 \%$ \\
\hline fr_sequoia & $81.12 \%$ & $71.34 \%$ & $74.41 \%$ & $83.39 \%$ & $73.59 \%$ & $69.28 \%$ & $2.27 \%$ & $2.25 \%$ & $-5.13 \%$ \\
\hline fr_spoken & $65.56 \%$ & $53.46 \%$ & $54.67 \%$ & $65.63 \%$ & $52.96 \%$ & $52.82 \%$ & $0.07 \%$ & $-0.50 \%$ & $-1.85 \%$ \\
\hline gl_ctg & $76.10 \%$ & $62.11 \%$ & $65.29 \%$ & $80.38 \%$ & $67.42 \%$ & $71.64 \%$ & $4.28 \%$ & $5.31 \%$ & $6.35 \%$ \\
\hline gl_treegal & $66.16 \%$ & $49.13 \%$ & $51.60 \%$ & $68.08 \%$ & $50.06 \%$ & $52.80 \%$ & $1.92 \%$ & $0.93 \%$ & $1.20 \%$ \\
\hline de_gsd & $70.85 \%$ & $34.09 \%$ & $60.56 \%$ & $71.88 \%$ & $35.12 \%$ & $34.30 \%$ & $1.03 \%$ & $1.03 \%$ & $-26.26 \%$ \\
\hline got_proiel & $62.16 \%$ & $48.57 \%$ & $55.02 \%$ & $65.49 \%$ & $51.72 \%$ & $54.63 \%$ & $3.33 \%$ & $3.15 \%$ & $-0.39 \%$ \\
\hline el_gdt & $82.11 \%$ & $65.33 \%$ & $68.67 \%$ & $82.56 \%$ & $65.58 \%$ & $64.68 \%$ & $0.45 \%$ & $0.25 \%$ & $-3.99 \%$ \\
\hline he_htb & $57.86 \%$ & $44.09 \%$ & $46.51 \%$ & $58.87 \%$ & $44.89 \%$ & $47.37 \%$ & $1.01 \%$ & $0.80 \%$ & $0.86 \%$ \\
\hline hi_hdtb & $87.15 \%$ & $69.09 \%$ & $79.93 \%$ & $88.43 \%$ & $70.48 \%$ & $81.52 \%$ & $1.28 \%$ & $1.39 \%$ & $1.59 \%$ \\
\hline hu_szeged & $66.76 \%$ & $52.82 \%$ & $56.92 \%$ & $68.74 \%$ & $54.66 \%$ & $53.52 \%$ & $1.98 \%$ & $1.84 \%$ & $-3.40 \%$ \\
\hline zh_gsd & $57.91 \%$ & $48.49 \%$ & $52.92 \%$ & $60.13 \%$ & $49.17 \%$ & $54.29 \%$ & $2.22 \%$ & $0.68 \%$ & $1.37 \%$ \\
\hline id_gsd & $74.37 \%$ & $63.42 \%$ & $62.50 \%$ & $75.51 \%$ & $63.54 \%$ & $71.50 \%$ & $1.14 \%$ & $0.12 \%$ & $9.00 \%$ \\
\hline ga_idt & $62.93 \%$ & $37.66 \%$ & $42.06 \%$ & $64.87 \%$ & $39.22 \%$ & $42.44 \%$ & $1.94 \%$ & $1.56 \%$ & $0.38 \%$ \\
\hline it_isdt & $86.26 \%$ & $77.06 \%$ & $77.12 \%$ & $88.28 \%$ & $79.48 \%$ & $72.47 \%$ & $2.02 \%$ & $2.42 \%$ & $-4.65 \%$ \\
\hline it_postwita & $66.81 \%$ & $53.64 \%$ & $53.99 \%$ & $67.58 \%$ & $53.93 \%$ & $44.53 \%$ & $0.77 \%$ & $0.29 \%$ & $-9.46 \%$ \\
\hline ja_gsd & $72.32 \%$ & $58.35 \%$ & $60.17 \%$ & $73.16 \%$ & $59.39 \%$ & $60.92 \%$ & $0.84 \%$ & $1.04 \%$ & $0.75 \%$ \\
\hline ja_modern & $22.71 \%$ & $10.00 \%$ & $10.00 \%$ & $22.71 \%$ & $10.00 \%$ & $10.00 \%$ & $0.00 \%$ & $0.00 \%$ & $0.00 \%$ \\
\hline kk_ktb & $24.21 \%$ & $10.00 \%$ & $10.00 \%$ & $24.21 \%$ & $10.00 \%$ & $10.00 \%$ & $0.00 \%$ & $0.00 \%$ & $0.00 \%$ \\
\hline ko_gsd & $61.40 \%$ & $54.10 \%$ & $50.50 \%$ & $74.94 \%$ & $68.34 \%$ & $62.21 \%$ & $13.54 \%$ & $14.24 \%$ & $11.71 \%$ \\
\hline ko_kaist & $70.25 \%$ & $61.49 \%$ & $57.68 \%$ & $82.74 \%$ & $75.55 \%$ & $69.47 \%$ & $12.49 \%$ & $14.06 \%$ & $11.79 \%$ \\
\hline kmr_mg & $23.92 \%$ & $5.00 \%$ & $11.86 \%$ & $23.92 \%$ & $5.00 \%$ & $11.86 \%$ & $0.00 \%$ & $0.00 \%$ & $0.00 \%$ \\
\hline la_ittb & $75.95 \%$ & $66.08 \%$ & $71.87 \%$ & $80.07 \%$ & $71.95 \%$ & $76.29 \%$ & $4.12 \%$ & $5.87 \%$ & $4.42 \%$ \\
\hline la_perseus & $47.61 \%$ & $30.16 \%$ & $32.19 \%$ & $49.99 \%$ & $31.35 \%$ & $33.75 \%$ & $2.38 \%$ & $1.19 \%$ & $1.56 \%$ \\
\hline la_proiel & $59.66 \%$ & $47.05 \%$ & $53.65 \%$ & $63.93 \%$ & $51.19 \%$ & $54.64 \%$ & $4.27 \%$ & $4.14 \%$ & $0.99 \%$ \\
\hline lv_lvtb & $69.43 \%$ & $54.96 \%$ & $58.25 \%$ & $70.89 \%$ & $56.14 \%$ & $57.30 \%$ & $1.46 \%$ & $1.18 \%$ & $-0.95 \%$ \\
\hline pcm_nsc & $12.18 \%$ & $5.00 \%$ & $10.87 \%$ & $10.00 \%$ & $5.00 \%$ & $5.00 \%$ & $-2.18 \%$ & $0.00 \%$ & $-5.87 \%$ \\
\hline sme_giella & $56.98 \%$ & $46.05 \%$ & $42.35 \%$ & $61.58 \%$ & $49.88 \%$ & $44.19 \%$ & $4.60 \%$ & $3.83 \%$ & $1.84 \%$ \\
\hline no_bokmaal & $83.47 \%$ & $74.65 \%$ & $76.32 \%$ & $85.29 \%$ & $76.97 \%$ & $70.82 \%$ & $1.82 \%$ & $2.32 \%$ & $-5.50 \%$ \\
\hline no_nynorsk & $82.13 \%$ & $72.40 \%$ & $74.22 \%$ & $84.09 \%$ & $74.71 \%$ & $69.97 \%$ & $1.96 \%$ & $2.31 \%$ & $-4.25 \%$ \\
\hline no_nynorsklia & $48.95 \%$ & $37.60 \%$ & $40.69 \%$ & $52.84 \%$ & $40.67 \%$ & $43.70 \%$ & $3.89 \%$ & $3.07 \%$ & $3.01 \%$ \\
\hline fro_srcmf & $79.27 \%$ & $70.70 \%$ & $74.45 \%$ & $82.70 \%$ & $75.06 \%$ & $78.96 \%$ & $3.43 \%$ & $4.36 \%$ & $4.51 \%$ \\
\hline cu_proiel & $65.46 \%$ & $53.96 \%$ & $58.39 \%$ & $70.03 \%$ & $58.51 \%$ & $63.28 \%$ & $4.57 \%$ & $4.55 \%$ & $4.89 \%$ \\
\hline fa_seraji & $79.10 \%$ & $72.20 \%$ & $69.43 \%$ & $79.57 \%$ & $71.96 \%$ & $69.42 \%$ & $0.47 \%$ & $-0.24 \%$ & $-0.01 \%$ \\
\hline pl_lfg & $87.53 \%$ & $74.54 \%$ & $78.58 \%$ & $88.78 \%$ & $75.92 \%$ & $77.55 \%$ & $1.25 \%$ & $1.38 \%$ & $-1.03 \%$ \\
\hline pl_sz & $81.90 \%$ & $63.84 \%$ & $71.98 \%$ & $83.54 \%$ & $65.25 \%$ & $72.25 \%$ & $1.64 \%$ & $1.41 \%$ & $0.27 \%$ \\
\hline pt_bosque & $82.07 \%$ & $67.40 \%$ & $72.04 \%$ & $84.59 \%$ & $70.21 \%$ & $62.91 \%$ & $2.52 \%$ & $2.81 \%$ & $-9.13 \%$ \\
\hline ro_rrt & $80.27 \%$ & $71.48 \%$ & $71.87 \%$ & $82.67 \%$ & $74.11 \%$ & $71.11 \%$ & $2.40 \%$ & $2.63 \%$ & $-0.76 \%$ \\
\hline ru_syntagrus & $84.59 \%$ & $76.87 \%$ & $78.01 \%$ & $87.70 \%$ & $79.58 \%$ & $82.35 \%$ & $3.11 \%$ & $2.71 \%$ & $4.34 \%$ \\
\hline ru_taiga & $55.51 \%$ & $36.79 \%$ & $39.79 \%$ & $57.94 \%$ & $38.59 \%$ & $42.12 \%$ & $2.43 \%$ & $1.80 \%$ & $2.33 \%$ \\
\hline sr_set & $82.07 \%$ & $70.04 \%$ & $74.12 \%$ & $83.54 \%$ & $70.86 \%$ & $66.69 \%$ & $1.47 \%$ & $0.82 \%$ & $-7.43 \%$ \\
\hline sk_snk & $75.41 \%$ & $54.38 \%$ & $60.35 \%$ & $78.45 \%$ & $56.57 \%$ & $67.75 \%$ & $3.04 \%$ & $2.19 \%$ & $7.40 \%$ \\
\hline sl_ssj & $77.33 \%$ & $63.47 \%$ & $68.93 \%$ & $79.15 \%$ & $65.05 \%$ & $69.10 \%$ & $1.82 \%$ & $1.58 \%$ & $0.17 \%$ \\
\hline sl_sst & $46.95 \%$ & $34.19 \%$ & $38.73 \%$ & $46.19 \%$ & $33.61 \%$ & $38.00 \%$ & $-0.76 \%$ & $-0.58 \%$ & $-0.73 \%$ \\
\hline es_ancora & $84.43 \%$ & $76.01 \%$ & $76.43 \%$ & $87.66 \%$ & $80.08 \%$ & $68.03 \%$ & $3.23 \%$ & $4.07 \%$ & $-8.40 \%$ \\
\hline sv_lines & $74.06 \%$ & $58.62 \%$ & $66.39 \%$ & $75.87 \%$ & $59.96 \%$ & $64.81 \%$ & $1.81 \%$ & $1.34 \%$ & $-1.58 \%$ \\
\hline sv_pud & $70.63 \%$ & $43.38 \%$ & $54.47 \%$ & $72.26 \%$ & $44.27 \%$ & $52.52 \%$ & $1.63 \%$ & $0.89 \%$ & $-1.95 \%$ \\
\hline sv_talbanken & $77.91 \%$ & $69.22 \%$ & $70.01 \%$ & $80.00 \%$ & $70.66 \%$ & $71.49 \%$ & $2.09 \%$ & $1.44 \%$ & $1.48 \%$ \\
\hline th_pud & $0.70 \%$ & $0.03 \%$ & $0.42 \%$ & $0.70 \%$ & $0.03 \%$ & $0.42 \%$ & $0.00 \%$ & $0.00 \%$ & $0.00 \%$ \\
\hline tr_imst & $54.04 \%$ & $44.50 \%$ & $45.91 \%$ & $57.57 \%$ & $46.59 \%$ & $46.27 \%$ & $3.53 \%$ & $2.09 \%$ & $0.36 \%$ \\
\hline uk_iu & $74.91 \%$ & $56.78 \%$ & $63.72 \%$ & $76.27 \%$ & $57.66 \%$ & $63.11 \%$ & $1.36 \%$ & $0.88 \%$ & $-0.61 \%$ \\
\hline hsb_ufal & $23.64 \%$ & $5.00 \%$ & $11.72 \%$ & $29.92 \%$ & $10.00 \%$ & $15.16 \%$ & $6.28 \%$ & $5.00 \%$ & $3.44 \%$ \\
\hline ur_udtb & $77.29 \%$ & $50.31 \%$ & $63.74 \%$ & $77.91 \%$ & $50.78 \%$ & $64.30 \%$ & $0.62 \%$ & $0.47 \%$ & $0.56 \%$ \\
\hline ug_udt & $56.26 \%$ & $36.82 \%$ & $43.53 \%$ & $55.88 \%$ & $35.84 \%$ & $43.16 \%$ & $-0.38 \%$ & $-0.98 \%$ & $-0.37 \%$ \\
\hline vi_vtb & $39.63 \%$ & $33.49 \%$ & $35.72 \%$ & $39.53 \%$ & $32.33 \%$ & $32.07 \%$ & $-0.10 \%$ & $-1.16 \%$ & $-3.65 \%$ \\
\hline
\end{tabular}

Table 5: All results on test set. 


\begin{tabular}{|c|c|c|c|c|c|c|c|c|c|}
\hline & \multicolumn{3}{|c|}{ Baseline } & \multicolumn{3}{|c|}{ Fudan } & \multicolumn{3}{|c|}{ Improvement } \\
\hline Language code & LAS & MLAS & BLEX & LAS & MLAS & BLEX & LAS & MLAS & BLEX \\
\hline el_gdt & $81.37 \%$ & $63.92 \%$ & $65.21 \%$ & $82.31 \%$ & $64.62 \%$ & $61.66 \%$ & $0.94 \%$ & $0.70 \%$ & $-3.55 \%$ \\
\hline tr_imst & $54.83 \%$ & $44.25 \%$ & $45.81 \%$ & $58.65 \%$ & $46.16 \%$ & $45.81 \%$ & $3.82 \%$ & $1.91 \%$ & $0.00 \%$ \\
\hline id_gsd & $74.40 \%$ & $63.51 \%$ & $63.29 \%$ & $74.82 \%$ & $63.16 \%$ & $71.21 \%$ & $0.42 \%$ & $-0.35 \%$ & $7.92 \%$ \\
\hline da_ddt & $75.16 \%$ & $65.29 \%$ & $66.07 \%$ & $78.20 \%$ & $68.34 \%$ & $63.80 \%$ & $3.04 \%$ & $3.05 \%$ & $-2.27 \%$ \\
\hline et_edt & $76.50 \%$ & $68.27 \%$ & $64.17 \%$ & $79.86 \%$ & $71.38 \%$ & $62.30 \%$ & $3.36 \%$ & $3.11 \%$ & $-1.87 \%$ \\
\hline got_proiel & $62.03 \%$ & $48.16 \%$ & $54.39 \%$ & $75.09 \%$ & $61.20 \%$ & $63.54 \%$ & $13.06 \%$ & $13.04 \%$ & $9.15 \%$ \\
\hline sl_ssj & $77.72 \%$ & $63.96 \%$ & $68.97 \%$ & $83.77 \%$ & $69.66 \%$ & $72.37 \%$ & $6.05 \%$ & $5.70 \%$ & $3.40 \%$ \\
\hline en_gum & $76.63 \%$ & $65.57 \%$ & $67.20 \%$ & $78.81 \%$ & $67.61 \%$ & $62.09 \%$ & $2.18 \%$ & $2.04 \%$ & $-5.11 \%$ \\
\hline cu_proiel & $66.12 \%$ & $54.48 \%$ & $59.16 \%$ & $79.39 \%$ & $67.52 \%$ & $70.80 \%$ & $13.27 \%$ & $13.04 \%$ & $11.64 \%$ \\
\hline ur_udtb & $77.44 \%$ & $49.91 \%$ & $63.55 \%$ & $77.92 \%$ & $50.79 \%$ & $64.01 \%$ & $0.48 \%$ & $0.88 \%$ & $0.46 \%$ \\
\hline fro_srcmf & $79.15 \%$ & $70.43 \%$ & $74.27 \%$ & $81.90 \%$ & $74.01 \%$ & $77.87 \%$ & $2.75 \%$ & $3.58 \%$ & $3.60 \%$ \\
\hline hi_hdtb & $87.26 \%$ & $69.78 \%$ & $80.59 \%$ & $88.55 \%$ & $71.17 \%$ & $82.16 \%$ & $1.29 \%$ & $1.39 \%$ & $1.57 \%$ \\
\hline ko_gsd & $57.25 \%$ & $49.06 \%$ & $44.24 \%$ & $72.41 \%$ & $65.18 \%$ & $57.04 \%$ & $15.16 \%$ & $16.12 \%$ & $12.80 \%$ \\
\hline cs_fictree & $83.16 \%$ & $70.72 \%$ & $75.80 \%$ & $85.99 \%$ & $73.49 \%$ & $77.82 \%$ & $2.83 \%$ & $2.77 \%$ & $2.02 \%$ \\
\hline gl_ctg & $76.32 \%$ & $62.58 \%$ & $65.57 \%$ & $81.75 \%$ & $68.93 \%$ & $73.42 \%$ & $5.43 \%$ & $6.35 \%$ & $7.85 \%$ \\
\hline lv_lvtb & $70.67 \%$ & $57.79 \%$ & $60.96 \%$ & $0.00 \%$ & $0.00 \%$ & $0.00 \%$ & $0.00 \%$ & $0.00 \%$ & $0.00 \%$ \\
\hline fr_gsd & $85.81 \%$ & $77.80 \%$ & $79.16 \%$ & $88.83 \%$ & $81.24 \%$ & $70.55 \%$ & $3.02 \%$ & $3.44 \%$ & $-8.61 \%$ \\
\hline ru_syntagrus & $83.87 \%$ & $75.78 \%$ & $77.27 \%$ & $0.00 \%$ & $0.00 \%$ & $0.00 \%$ & $0.00 \%$ & $0.00 \%$ & $0.00 \%$ \\
\hline hu_szeged & $68.41 \%$ & $56.47 \%$ & $60.17 \%$ & $70.67 \%$ & $58.33 \%$ & $57.56 \%$ & $2.26 \%$ & $1.86 \%$ & $-2.61 \%$ \\
\hline sv_lines & $76.23 \%$ & $62.16 \%$ & $67.63 \%$ & $77.91 \%$ & $63.39 \%$ & $65.60 \%$ & $1.68 \%$ & $1.23 \%$ & $-2.03 \%$ \\
\hline no_bokmaal & $84.56 \%$ & $75.95 \%$ & $78.04 \%$ & $86.54 \%$ & $78.64 \%$ & $72.31 \%$ & $1.98 \%$ & $2.69 \%$ & $-5.73 \%$ \\
\hline sv_talbanken & $75.39 \%$ & $66.87 \%$ & $68.25 \%$ & $0.00 \%$ & $0.00 \%$ & $0.00 \%$ & $0.00 \%$ & $0.00 \%$ & $0.00 \%$ \\
\hline es_ancora & $85.08 \%$ & $76.81 \%$ & $77.48 \%$ & $88.21 \%$ & $80.75 \%$ & $68.74 \%$ & $3.13 \%$ & $3.94 \%$ & $-8.74 \%$ \\
\hline he_htb & $61.95 \%$ & $49.28 \%$ & $51.45 \%$ & $79.89 \%$ & $65.68 \%$ & $67.50 \%$ & $17.94 \%$ & $16.40 \%$ & $16.05 \%$ \\
\hline uk_iu & $77.94 \%$ & $59.66 \%$ & $68.07 \%$ & $78.75 \%$ & $60.11 \%$ & $66.23 \%$ & $0.81 \%$ & $0.45 \%$ & $-1.84 \%$ \\
\hline grc_proiel & $69.13 \%$ & $52.42 \%$ & $57.92 \%$ & $77.67 \%$ & $61.50 \%$ & $59.95 \%$ & $8.54 \%$ & $9.08 \%$ & $2.03 \%$ \\
\hline eu_bdt & $70.06 \%$ & $57.46 \%$ & $63.39 \%$ & $72.94 \%$ & $59.11 \%$ & $56.95 \%$ & $2.88 \%$ & $1.65 \%$ & $-6.44 \%$ \\
\hline fi_ftb & $75.76 \%$ & $65.72 \%$ & $62.68 \%$ & $79.90 \%$ & $70.61 \%$ & $69.82 \%$ & $4.14 \%$ & $4.89 \%$ & $7.14 \%$ \\
\hline cs_pdt & $84.85 \%$ & $75.35 \%$ & $80.55 \%$ & $86.83 \%$ & $77.45 \%$ & $75.86 \%$ & $1.98 \%$ & $2.10 \%$ & $-4.69 \%$ \\
\hline sk_snk & $75.73 \%$ & $54.34 \%$ & $59.71 \%$ & $80.35 \%$ & $57.64 \%$ & $69.80 \%$ & $4.62 \%$ & $3.30 \%$ & $10.09 \%$ \\
\hline hr_set & $77.84 \%$ & $59.60 \%$ & $69.99 \%$ & $80.63 \%$ & $61.89 \%$ & $66.75 \%$ & $2.79 \%$ & $2.29 \%$ & $-3.24 \%$ \\
\hline no_nynorsk & $82.75 \%$ & $73.88 \%$ & $75.76 \%$ & $85.07 \%$ & $76.64 \%$ & $72.06 \%$ & $2.32 \%$ & $2.76 \%$ & $-3.70 \%$ \\
\hline grc_perseus & $57.89 \%$ & $30.80 \%$ & $40.49 \%$ & $63.21 \%$ & $34.03 \%$ & $39.99 \%$ & $5.32 \%$ & $3.23 \%$ & $-0.50 \%$ \\
\hline fr_spoken & $65.09 \%$ & $54.00 \%$ & $55.42 \%$ & $73.46 \%$ & $64.24 \%$ & $63.19 \%$ & $8.37 \%$ & $10.24 \%$ & $7.77 \%$ \\
\hline pl_sz & $82.65 \%$ & $63.92 \%$ & $72.59 \%$ & $84.02 \%$ & $65.11 \%$ & $73.04 \%$ & $1.37 \%$ & $1.19 \%$ & $0.45 \%$ \\
\hline fi_tdt & $76.39 \%$ & $68.60 \%$ & $62.33 \%$ & $0.00 \%$ & $0.00 \%$ & $0.00 \%$ & $0.00 \%$ & $0.00 \%$ & $0.00 \%$ \\
\hline ca_ancora & $85.63 \%$ & $77.04 \%$ & $77.56 \%$ & $88.30 \%$ & $80.31 \%$ & $67.64 \%$ & $2.67 \%$ & $3.27 \%$ & $-9.92 \%$ \\
\hline ar_padt & $66.81 \%$ & $55.67 \%$ & $57.90 \%$ & $76.11 \%$ & $63.98 \%$ & $65.94 \%$ & $9.30 \%$ & $8.31 \%$ & $8.04 \%$ \\
\hline sr_set & $82.12 \%$ & $69.12 \%$ & $73.06 \%$ & $84.49 \%$ & $70.73 \%$ & $67.66 \%$ & $2.37 \%$ & $1.61 \%$ & $-5.40 \%$ \\
\hline bg_btb & $84.67 \%$ & $74.54 \%$ & $73.78 \%$ & $86.73 \%$ & $76.96 \%$ & $78.24 \%$ & $2.06 \%$ & $2.42 \%$ & $4.46 \%$ \\
\hline vi_vtb & $43.65 \%$ & $37.39 \%$ & $39.18 \%$ & $57.34 \%$ & $49.57 \%$ & $47.08 \%$ & $13.69 \%$ & $12.18 \%$ & $7.90 \%$ \\
\hline$\overline{d e \_g s d}$ & $75.55 \%$ & $38.52 \%$ & $65.39 \%$ & $77.00 \%$ & $39.91 \%$ & $40.77 \%$ & $1.45 \%$ & $1.39 \%$ & $-24.62 \%$ \\
\hline fr_seguoia & $82.72 \%$ & $74.13 \%$ & $76.34 \%$ & $85.71 \%$ & $76.53 \%$ & $71.16 \%$ & $2.99 \%$ & $2.40 \%$ & $-5.18 \%$ \\
\hline cs_cac & $84.42 \%$ & $72.17 \%$ & $78.29 \%$ & $86.46 \%$ & $74.65 \%$ & $77.70 \%$ & $2.04 \%$ & $2.48 \%$ & $-0.59 \%$ \\
\hline pl_lfg & $88.79 \%$ & $75.15 \%$ & $79.18 \%$ & $89.98 \%$ & $76.88 \%$ & $79.01 \%$ & $1.19 \%$ & $1.73 \%$ & $-0.17 \%$ \\
\hline en_lines & $75.78 \%$ & $66.29 \%$ & $68.57 \%$ & $78.17 \%$ & $67.59 \%$ & $67.22 \%$ & $2.39 \%$ & $1.30 \%$ & $-1.35 \%$ \\
\hline zh_gsd & $57.39 \%$ & $48.19 \%$ & $52.84 \%$ & $70.09 \%$ & $58.37 \%$ & $64.74 \%$ & $12.70 \%$ & $10.18 \%$ & $11.90 \%$ \\
\hline it_postwita & $65.85 \%$ & $52.14 \%$ & $52.90 \%$ & $77.23 \%$ & $66.00 \%$ & $53.42 \%$ & $11.38 \%$ & $13.86 \%$ & $0.52 \%$ \\
\hline la_proiel & $61.33 \%$ & $48.40 \%$ & $55.10 \%$ & $74.41 \%$ & $61.54 \%$ & $64.39 \%$ & $13.08 \%$ & $13.14 \%$ & $9.29 \%$ \\
\hline fa_seraji & $79.78 \%$ & $73.03 \%$ & $73.35 \%$ & $80.41 \%$ & $72.61 \%$ & $73.63 \%$ & $0.63 \%$ & $-0.42 \%$ & $0.28 \%$ \\
\hline af_afribooms & $80.19 \%$ & $65.98 \%$ & $70.40 \%$ & $80.95 \%$ & $67.26 \%$ & $68.10 \%$ & $0.76 \%$ & $1.28 \%$ & $-2.30 \%$ \\
\hline ko_kaist & $71.00 \%$ & $63.32 \%$ & $59.19 \%$ & $83.17 \%$ & $77.37 \%$ & $71.51 \%$ & $12.17 \%$ & $14.05 \%$ & $12.32 \%$ \\
\hline la_ittb & $73.23 \%$ & $59.94 \%$ & $67.43 \%$ & $78.06 \%$ & $66.21 \%$ & $72.02 \%$ & $4.83 \%$ & $6.27 \%$ & $4.59 \%$ \\
\hline en_ewt & $77.62 \%$ & $68.58 \%$ & $70.98 \%$ & $0.00 \%$ & $0.00 \%$ & $0.00 \%$ & $0.00 \%$ & $0.00 \%$ & $0.00 \%$ \\
\hline ug_udt & $56.88 \%$ & $37.43 \%$ & $43.34 \%$ & $57.78 \%$ & $37.54 \%$ & $44.08 \%$ & $0.90 \%$ & $0.11 \%$ & $0.74 \%$ \\
\hline pt_bosque & $84.93 \%$ & $73.22 \%$ & $76.02 \%$ & $88.19 \%$ & $76.65 \%$ & $66.58 \%$ & $3.26 \%$ & $3.43 \%$ & $-9.44 \%$ \\
\hline ro_rrt & $80.32 \%$ & $71.21 \%$ & $71.82 \%$ & $83.42 \%$ & $74.27 \%$ & $71.73 \%$ & $3.10 \%$ & $3.06 \%$ & $-0.09 \%$ \\
\hline nl_lassysmall & $73.61 \%$ & $59.99 \%$ & $61.71 \%$ & $77.63 \%$ & $64.44 \%$ & $52.55 \%$ & $4.02 \%$ & $4.45 \%$ & $-9.16 \%$ \\
\hline it_isdt & $85.95 \%$ & $77.20 \%$ & $77.37 \%$ & $87.81 \%$ & $79.30 \%$ & $71.82 \%$ & $1.86 \%$ & $2.10 \%$ & $-5.55 \%$ \\
\hline nl_alpino & $80.21 \%$ & $67.14 \%$ & $69.77 \%$ & $81.66 \%$ & $69.04 \%$ & $58.68 \%$ & $1.45 \%$ & $1.90 \%$ & $-11.09 \%$ \\
\hline ja_gsd & $75.48 \%$ & $62.39 \%$ & $64.58 \%$ & $92.51 \%$ & $83.13 \%$ & $85.11 \%$ & $17.03 \%$ & $20.74 \%$ & $20.53 \%$ \\
\hline
\end{tabular}

Table 6: All results on development set. 


\section{References}

Mathieu Dehouck and Pascal Denis. 2017. Delexicalized word embeddings for cross-lingual dependency parsing. In EACL. hal.inria.fr, volume 1, pages 241250 .

Timothy Dozat, Peng Qi, and Christopher D Manning. 2017. Stanford's graph-based neural dependency parser at the conll 2017 shared task. Proceedings of the CoNLL 2017 Shared Task: Multilingual Parsing from Raw Text to Universal Dependencies pages 20-30.

Long Duong, Trevor Cohn, Steven Bird, and Paul Cook. 2015. Low resource dependency parsing: Cross-lingual parameter sharing in a neural network parser. In Proceedings of the 53rd Annual Meeting of the Association for Computational Linguistics and the 7th International Joint Conference on Natural Language Processing (Volume 2: Short Papers). volume 2, pages 845-850.

Yarin Gal and Zoubin Ghahramani. 2016. A theoretically grounded application of dropout in recurrent neural networks. In Advances in neural information processing systems. pages 1019-1027.

Jiang Guo, Wanxiang Che, David Yarowsky, Haifeng Wang, and Ting Liu. 2015. Cross-lingual dependency parsing based on distributed representations. In Proceedings of the 53rd Annual Meeting of the Association for Computational Linguistics and the 7th International Joint Conference on Natural Language Processing (Volume 1: Long Papers). volume 1, pages 1234-1244.

Joakim Nivre et al. 2018. Universal Dependencies 2.2. LINDAT/CLARIN digital library at the Institute of Formal and Applied Linguistics, Charles University, Prague, http: //hdl.handle.net/11234/1-1983xxx. http://hdl.handle.net/11234/1-1983xxx.

Kenji Sagae and Alon Lavie. 2006. Parser combination by reparsing. In Proceedings of the Human Language Technology Conference of the NAACL, Companion Volume: Short Papers. Association for Computational Linguistics, pages 129-132.

Motoki Sato, Hitoshi Manabe, Hiroshi Noji, and Yuji Matsumoto. 2017. Adversarial training for crossdomain universal dependency parsing. In Proceedings of the CoNLL 2017 Shared Task: Multilingual Parsing from Raw Text to Universal Dependencies. Association for Computational Linguistics, pages 71-79. https://doi.org/10.18653/v1/K17-3007.

Tianze Shi, Felix G. Wu, Xilun Chen, and Yao Cheng. 2017. Combining global models for parsing universal dependencies. In Proceedings of the CoNLL 2017 Shared Task: Multilingual Parsing from Raw Text to Universal Dependencies. Association for Computational Linguistics, pages 31-39. https://doi.org/10.18653/v1/K17-3003.
Milan Straka, Jan Hajič, and Jana Straková. 2016. UDPipe: trainable pipeline for processing CoNLL-U files performing tokenization, morphological analysis, POS tagging and parsing. In Proceedings of the 10th International Conference on Language Resources and Evaluation (LREC 2016). European Language Resources Association, Portoro, Slovenia.

Milan Straka and Jana Straková. 2017. Tokenizing, pos tagging, lemmatizing and parsing ud 2.0 with udpipe. In Proceedings of the CoNLL 2017 Shared Task: Multilingual Parsing from Raw Text to Universal Dependencies. Association for Computational Linguistics, Vancouver, Canada, pages 8899. http://www.aclweb.org/anthology/K/K17/K173009.pdf.

Clara Vania, Xingxing Zhang, and Adam Lopez. 2017. Uparse: the edinburgh system for the conll 2017 ud shared task. In Proceedings of the CoNLL 2017 Shared Task: Multilingual Parsing from Raw Text to Universal Dependencies. Association for Computational Linguistics, pages 100-110. https://doi.org/10.18653/v1/K17-3010.

Dan Zeman et al. 2018a. Universal Dependencies 2.2 CoNLL 2018 shared task development and test data. LINDAT/CLARIN digital library at the Institute of Formal and Applied Linguistics, Charles University, Prague, http://hdl.handle.net/ 11234/1-2184. http://hdl.handle.net/11234/12184.

Daniel Zeman, Filip Ginter, Jan Hajič, Joakim Nivre, Martin Popel, Milan Straka, and et al. 2018b. CoNLL 2018 Shared Task: Multilingual Parsing from Raw Text to Universal Dependencies. In Proceedings of the CoNLL 2018 Shared Task: Multilingual Parsing from Raw Text to Universal Dependencies. Association for Computational Linguistics, pages 1-20.

Barret Zoph and Quoc V Le. 2016. Neural architecture search with reinforcement learning. arXiv preprint arXiv: 1611.01578 . 